\title{
Optimal Sensor Configuration and Fault-Tolerant Estimation of Vehicle States
}

\author{
Reza Zarringhalam and Ayyoub Rezaeian \\ Univ. of Waterloo \\ Saber Fallah \\ Univ. of Surrey \\ Amir Khajepour and William Melek \\ Univ. of Waterloo \\ Shih-Ken Chen and Baktiarr Litkouhi \\ General Motors Company
}

\begin{abstract}
This paper discusses observability of the vehicle states using different sensor configurations as well as fault-tolerant estimation of these states. The optimality of the sensor configurations is assessed through different observability measures and by using a 3-DOF linear vehicle model that incorporates yaw, roll and lateral motions of the vehicle. The most optimal sensor configuration is adopted and an observer is designed to estimate the states of the vehicle handling dynamics. Robustness of the observer against sensor failure is investigated. A fault-tolerant adaptive estimation algorithm is developed to mitigate any possible faults arising from the sensor failures. Effectiveness of the proposed fault-tolerant estimation scheme is demonstrated through numerical analysis and CarSim simulation.
\end{abstract}

CITATION: Zarringhalam, R., Rezaeian, A., Fallah, S., Khajepour, A. et al., "Optimal Sensor Configuration and FaultTolerant Estimation of Vehicle States," SAE Int. J. Passeng. Cars - Electron. Electr. Syst. 6(1):2013, doi:

10.4271/2013-01-0175.

\section{INTRODUCTION}

Reliability of sensory measurements or estimated states is a major concern in automotive applications. Many advanced vehicle subsystems such as stability controllers and $\mathrm{x}$-by-wire structures rely on the sensory measurements or estimated information. Noticeably, malfunction of such systems may cause unexpected consequences if not properly handled by the vehicle system. Installation of redundant sensors may help to guarantee desirable performance even with sensor failures. However, the number and type of the sensors for estimation or control purposes are important in the vehicle manufacture due to cost and performance. Thus, achieving observability and fault-tolerant estimation using a minimal and reliable sensor set is among the most important issues for the automotive suppliers and OEMs.

Literature suggests different approaches for observability analysis and determination of optimal sensor configuration.
Measuring the degree of observability is widely used to find the optimal sensor configuration. A set of sensors that results in the highest degree of observability, among all of the possible sensor sets, is considered as the optimal configuration for the system. There are some criteria to measure the degree of observability. For example, Muller and Weber [1] outlined three candidate measures including minimum eigenvalue of the observability Gramian to find the degree of observability for linear systems. Chamseddine et al. in [2] introduced two other criteria: the trace of the observability Gramian and the robust spectral norm which is the minimum singular value of the observability Gramian. In fact, these criteria are scalar numbers which provide a measure of how far the system with the current sensor configuration is from being unobservable.

Sumana and Venkateswarlu [3] used the trace of the observability Gramian and the condition number to obtain the best sensor configuration for a reactive distillation column. 
The set with the maximum trace or minimum condition number was chosen as the optimal sensor configuration. Nakhaeinejad et al. [4] suggested a similar approach not only for analysis of the faults, but also for defining a guideline for model-based sensor selection for induction motors. Among all of the abovementioned methods, the condition number is more common and is frequently used in the literature [1], [ $\underline{5}]$, [6]. In this paper, all of the introduced observability and sensor configuration methods are chosen and applied to determine the best sensor configuration for estimation of vehicle states. Such states are required by many automotive applications such as traction control system, ABS or other vehicle safety algorithms [].

Despite somewhat robustness against uncertainties, these estimation methods are still sensitive to the sensor failures. Faulty sensory signals or estimation can be problematic for a closed loop control systems that rely on these estimated or sensory signals, because the controller can guide the system away from the set point, and even to instability. Therefore, a need arises to develop intelligent methods to mitigate the effects of sensor failures on the control performance.

Metallidis et al. discussed the fault detection and optimal sensor location for vehicle suspension in []․ They presented a statistical framework to formulate the sensor location and fault detection problems as optimization problems. Kim and Lee [9] presented an analytical sensor fault detection algorithm for vertical accelerometers which are used as a part of a continuous damping control system of a vehicle. Fault detection for lateral and vertical dynamics of a vehicle was discussed in [10]. In this approach, roll dynamics and lateral load transfer are neglected.

Measured and estimated vehicle states are extensively used by many recent vehicle control and safety systems to ensure stability of the vehicle [11]-[12]. This paper studies optimal sensor configuration, sensor fault detection and faulttolerant estimation for vehicle handling dynamics. Contents of this paper are organized as follows: Section II introduces developing a 3-DOF vehicle handling model which is used as a basis for evaluation of the sensor sets and development of the fault-tolerant estimation scheme. The model is simulated in Matlab and validated with CarSim. Section III investigates optimal sensor configuration from a finite set of sensors commonly used for vehicle control applications. Observability and optimal sensor configuration measures are described and preference of the sensor sets is quantified. Section IV discusses fault-tolerant estimation of vehicle states. Additionally, the estimation process using the Luenberger observers and LQR gain tuning is described, simulation results are provided, and performance of the estimator for the cases that one of the vehicle sensors fails is simulated and analyzed. Moreover, development of a faulttolerant adaptive estimation algorithm to mitigate possible faults arising from sensor failures is described in this section. The reliable performance of the fault-tolerant estimation scheme is demonstrated through simulations. Finally, a conclusion is presented in Section $\mathrm{V}$.

\section{VEHICLE MODEL}

Sufficiently accurate modeling of the vehicle dynamics is the first step in developing estimation or fault detection methods. Vehicle state observability and fault-tolerant estimation of vehicle states are herein studied using a 3-DOF vehicle model shown in Figure 1. This model represents handling dynamics of the vehicle and includes lateral acceleration $\left(a_{y}\right)$, yaw rate (r) and roll rate $(\dot{\phi})$ as dominant elements that contribute to the handling performance. The majority of studies that focused on vehicle handling dynamics consider a 2-DOF vehicle model that only includes yaw and lateral motions [13], [14], [15]. However, the roll motion can significantly affect the handling dynamics due to lateral load transfer. Roll motion also affects dynamics of the steering and suspension systems [16]. Therefore, the 3-DOF model adopted in this paper can result in a better modeling accuracy which is an essential requirement for state estimation and sensor fault detection applications.

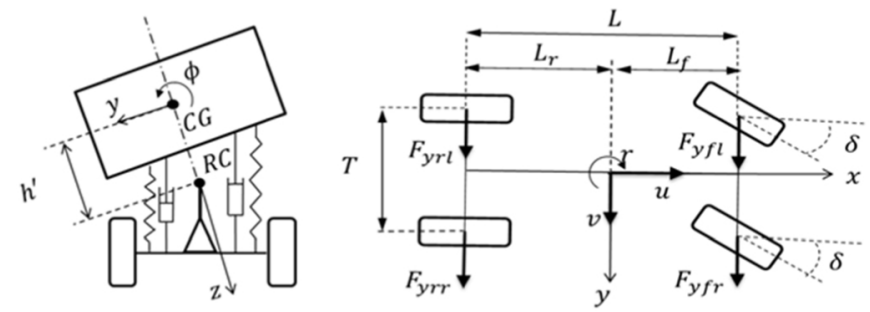

Figure 1. Vehicle model

The state-space representation of the model is formed as:

$$
\dot{X}=A X+B u
$$

where lateral velocity $\left(v_{y}\right)$, yaw rate $(r)$, roll angle $(\phi)$ and roll rate $(\dot{\phi})$ are selected as the states and steering angle $(\delta)$ is the input of the system:

$$
\begin{gathered}
X=\left[v_{y} r \phi \dot{\phi}\right]^{T} \\
u=\delta
\end{gathered}
$$

The system $(A)$ and input $(B)$ matrices are dependent on the geometrical and dynamic properties of the vehicle as well as characteristics of the suspension system, steering system, and tires. Considering vehicle lateral dynamics, roll dynamics, lateral load transfer, linear tire models, camber-byroll, steering-by-roll and scrub-by-roll properties, the system and measurement matrices of the handling dynamics are formed as 


$$
\begin{aligned}
& A=\left[\begin{array}{llll}
a_{11} & a_{12} & a_{13} & a_{14} \\
a_{21} & a_{22} & a_{23} & a_{24} \\
a_{31} & a_{32} & a_{33} & a_{34} \\
a_{41} & a_{42} & a_{43} & a_{44}
\end{array}\right] \\
& a_{11}=-2 \frac{C_{\alpha f}+C_{\alpha r}}{u . m} \\
& a_{21}=-2 \frac{L_{f} \cdot C_{\alpha f}-L_{r} \cdot C_{\alpha r}}{u . m} \\
& a_{31}=0 \\
& a_{41}=2 \frac{m_{s} h^{\prime}\left(C_{\alpha f}+C_{\alpha r}\right)}{m \cdot I_{x} \cdot u} \\
& a_{12}=-2 \frac{L_{f} \cdot C_{\alpha f}-L_{r} \cdot C_{\alpha r}}{u \cdot m}-u \\
& a_{22}=-2 \frac{L_{f}^{2} \cdot C_{\alpha f}+L_{r}^{2} \cdot C_{\alpha r}}{u . I_{Z}} \\
& a_{32}=0 \\
& a_{42}=\frac{m_{s} \cdot h^{\prime}}{m \cdot I_{x}}\left(2 \frac{L_{f} \cdot C_{\alpha f}-L_{r} \cdot C_{\alpha r}}{u}+u \cdot m\right)-\frac{m_{s} \cdot h^{\prime} \cdot u}{I x} \\
& a_{13}=\frac{2}{m}\left(C_{\alpha f} \cdot K_{f S B R}+C_{\alpha r} \cdot K_{r S B R}+C_{\gamma f} \cdot K_{f C B R}+\right. \\
& \left.C_{\gamma r} \cdot K_{r C B R}\right) \\
& a_{23}=\frac{2}{I_{z}}\left(L_{f} \cdot C_{\alpha f} \cdot K_{f S B R}-L_{r} \cdot C_{\alpha r} \cdot K_{r S B R}+\right. \\
& \left.L_{f} \cdot C_{\gamma f} \cdot K_{f C B R}-L_{r} \cdot C_{\gamma r} \cdot K_{r C B R}\right) \\
& a_{33}=0 \\
& a_{43}=\frac{m_{s} \cdot g \cdot h^{\prime}-K_{t}}{I_{x}}- \\
& \frac{m_{s} \cdot h^{\prime}\left(C_{\alpha f \cdot} \cdot K_{f S B R}+C_{\alpha r} \cdot K_{r S B R}+C_{\gamma f} \cdot K_{f C B R}+C_{\gamma r} \cdot K_{r C B R}\right)}{m \cdot I_{x} \cdot u} \\
& a_{14}=-2 \frac{C_{\alpha f \cdot K_{f S C B R}+C_{\alpha r} \cdot K_{r S C B R}}}{u . m} \\
& a_{24}=-2 \frac{L_{f} \cdot C_{\alpha f} \cdot K_{f S C B R}-L_{r} \cdot C_{\alpha r} \cdot K_{r S C B R}}{u \cdot I_{z}}
\end{aligned}
$$

$$
\begin{gathered}
a_{34}=1 \\
a_{44}=2 \frac{m_{s} \cdot h^{\prime}\left(c_{\alpha f \cdot} \cdot K_{f S C B R}+c_{\alpha r} \cdot K_{r S C B R}\right)}{m \cdot I_{x} \cdot u}-\frac{c_{t}}{I_{x}} \\
B=\left[\begin{array}{l}
b_{11} \\
b_{21} \\
b_{31} \\
b_{41}
\end{array}\right]=\left[\begin{array}{l}
2 \frac{c_{\alpha f}}{m} \\
2 \frac{L_{f} \cdot C_{\alpha f}}{I_{z}} \\
0 \\
\frac{m_{s} \cdot h^{\prime} \cdot c_{\alpha f}}{m \cdot I_{x}}
\end{array}\right]
\end{gathered}
$$

Definitions of the model parameters are provided in Definitions/Abbreviations Section.

The measurement equation for the system is also dependent on the vehicle parameters

$$
Y=C X+D u
$$

Depending on the type and locations of the vehicle sensors, the $C$ and $D$ matrices have different dimensions and magnitudes. For the case when lateral acceleration, yaw rate and, roll rat rate sensors are assumed available (i.e. $\left.Y=\left[a_{y}, r, \dot{\phi}\right]^{T}\right)$, the measurement matrices are

$$
C=\left[\begin{array}{cccc}
\frac{-2\left(c_{\alpha f}+C_{\alpha r}\right)}{u . m} & \frac{-2\left(L_{f} . C_{\alpha f}-L_{r} . C_{\alpha r}\right)}{u . m}+u & 0 & \frac{2 m_{s} h^{\prime}\left(c_{\alpha f}+C_{\alpha r}\right)}{m . I_{x} \cdot u} \\
0 & 1 & 0 & 0 \\
0 & 0 & 0 & 1
\end{array}\right]
$$

$$
D=\left[\begin{array}{c}
2 \frac{C_{\alpha f}}{m} \\
0 \\
0
\end{array}\right]
$$

The values of the model parameters are available for commercial vehicles through standard vehicle tests from the automotive manufacture.

Accuracy of the model is validated through CarSim simulations for different maneuvers. It should be noted that the introduced model is valid for linear regions of vehicle dynamics (i.e. $a_{y}<0.4 \mathrm{~g}$ ). Moreover, the model assumes a constant or slowly-varying longitudinal velocity. This can be justified noting that the handling dynamics is much faster compared to the longitudinal dynamics and quasi-linearity of the model is satisfied.

Figure 2 illustrates simulation results for a double-lanechange maneuver at $60 \mathrm{kph}$. The results verify accuracy of the model despite its simplicity. 

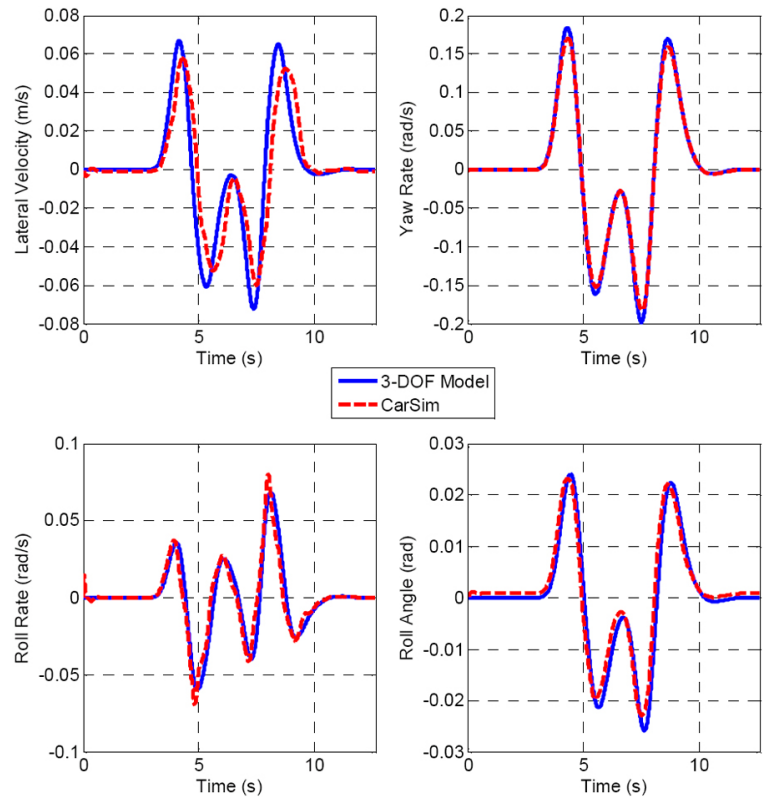

Figure 2. Verification of the 3-DOF model with CarSim

\section{OBSERVABILITY AND OPTIMAL SENSOR CONFIGURATION}

Selection of a minimum number (and optimal placement) of sensors to obtain the maximum amount of information for reliable state estimation is the key objective for the optimum sensor configuration.

In this paper, several observability measures are selected and applied to determine the best sensor configuration for estimation of vehicle states.

For the 3-DOF vehicle model described in Section II, the available sensors include yaw rate, roll rate and lateral acceleration sensors, which are measurable using an Inertial Measurement Unit (IMU) used in many modern vehicles. The 3-DOF vehicle model is a linear model that allows utilization of linear observability analysis measures. For a linear dynamic system described by Equations (1) and (22), the observability Gramian is calculated as [17]

$$
W_{O}=\int_{0}^{\infty} e^{A^{T} t} C^{T} C e^{A t} d t
$$

The system is observable if the observability Gramian is a full rank matrix

$$
r\left(W_{0}\right)=n
$$

where $r\left(W_{0}\right)$ represents the rank of the observability Gramian and $n$ is the number of states. In other words, the sensor set described by the matrix $C$ can generate enough information to reconstruct all of the states of the system if $W_{0}$ is rank sufficient. However, this information is not sufficient to assess the optimality of the sensor configuration. As discussed in the Introduction Section, literature suggests a variety of different measures to analyze optimality of a sensor set. For such analysis, seven different measures are considered in this paper:

- Minimum eigenvalue of the observability Gramian matrix:

$$
\mu_{1}=\lambda_{\min }\left(W_{O}\right)
$$

- Inverse trace of the inverse observability Gramian:

$$
\mu_{2}=\frac{n}{\operatorname{trace}\left(W_{O}^{-1}\right)}
$$

- Determinant of the observability Gramian:

$$
\mu_{3}=\left[\operatorname{det}\left(W_{O}\right)\right]^{\frac{1}{2}}
$$

- Condition number:

$$
C . N .=\frac{\sigma_{\max }\left(W_{O}\right)}{\sigma_{\min }\left(W_{O}\right)}
$$

- Smallest singular value of the observability Gramian:

$$
N . S .=\sigma_{\min }\left(W_{O}\right)
$$

- Spectral radius:

$$
\rho=\sigma_{\max }\left(W_{o}\right)
$$

- Trace of the observability Gramian:

$$
\operatorname{trace}\left(W_{O}\right)=\sum_{i=1}^{n} \sigma_{i}\left(W_{O}\right)
$$

In the above equations, $\lambda$ represents the eigenvalue and $\sigma$ represents the singular value. For detailed descriptions of the observability and optimal sensor configuration analysis, the reader is referred to [17]. Except for the condition number, a larger value of the measures indicates better reliability of the sensor set and observability of the system. For the condition number, a smaller value is more desirable. The results for assessment of all of the possible sensor sets for the 3-DOF model are presented in Table 1. 
Table 1. Results of the observability analysis

\begin{tabular}{|c|c|c|c|c|c|c|c|}
\hline \multirow{2}{*}{$\begin{array}{c}\text { Sensor } \\
\text { Set }\end{array}$} & \multicolumn{5}{|c|}{ Observability Criteria for Optimal Sensor Configuration } \\
\cline { 2 - 8 } & $\boldsymbol{\mu}_{1}$ & $\boldsymbol{\mu}_{2}$ & $\boldsymbol{\mu}_{3}$ & $\boldsymbol{C} . \boldsymbol{N}$. & $\boldsymbol{N} . \boldsymbol{S}$. & $\boldsymbol{\rho}$ & Trace \\
\hline$r$ & $5.57 \mathrm{E}-09$ & $2.22 \mathrm{E}-08$ & $1.84 \mathrm{E}-05$ & 5649220 & $5.57 \mathrm{E}-09$ & 0.031442 & 0.031786 \\
\hline$a_{y}$ & $3.07 \mathrm{E}-05$ & 0.000122 & 0.031727 & 237262.5 & $3.07 \mathrm{E}-05$ & 7.274732 & 7.95199 \\
\hline$\dot{\phi}$ & 0.001194 & 0.004329 & 0.051412 & 5113.608 & 0.001194 & 6.103245 & 6.186823 \\
\hline$a_{y}, r$ & $3.49 \mathrm{E}-05$ & 0.000139 & 0.033588 & 208748.2 & $3.49 \mathrm{E}-05$ & 7.285149 & 7.983776 \\
\hline$r, \dot{\phi}$ & 0.007628 & 0.023174 & 0.104928 & 800.0728 & 0.007628 & 6.103306 & 6.218609 \\
\hline$a_{y}, \dot{\phi}$ & 0.049408 & 0.181189 & 1.092958 & 154.6395 & 0.049408 & 7.640483 & 14.13881 \\
\hline$a_{y}, r, \dot{\phi}$ & 0.049414 & 0.181607 & 1.102197 & 154.7957 & 0.049414 & 7.649005 & 14.1706 \\
\hline
\end{tabular}

According to the results, the system is observable for all of the sensor sets introduced in Table 1, i.e., the observability Gramian is full rank for all of the cases. However, the results also imply that the sensor set that includes all of the available sensors represents the best sensor configuration for the system. Such an outcome is expected because this sensor set can deliver the most possible information about dynamics of the system. The second rank goes to the sensor set that includes lateral acceleration and roll rate sensors. The sensor set including roll rate and yaw rate ranks third, followed by the sensor set including lateral acceleration and yaw rate. Sensor sets including individual sensors are the least favorable configurations based on the measures in Equations (27), (28), (29), (30), (31), (32), (33). However, the system is observable with all the introduced sensor sets, assuming the model (1), (2), (3), (4), (5), (6), (7), (8), (9), (10), (11), (12), (13), (14), (15), (16), (17), (18), (19), (20), (21), (22), (23), $(24)$ is valid.

\section{FAULT TOLERANT ESTIMATION OF THE STATES}

\section{Estimation of Vehicle States}

The objective of the state estimation is to accurately reconstruct hard-to-measured states of a system despite model uncertainties and noisy sensory signals. Commonlyused vehicle sensors, such as lateral acceleration, roll rate, and yaw rate, are assumed as measurable states. Such measurements are available using a six-axis IMU. Moreover, the optimal sensor configuration analysis in Section III verified that this set is the optimal sensor configuration for the system. Therefore, other immeasurable states of the system (e.g. roll angle and lateral velocity) should be estimated using an observer. Linearity of the 3-DOF model enables application of the Luenberger observer as the estimation method. The observer gain $(L)$ is calculated using the LQR theory. Detailed description of the Luenberger observer design and LQR gain tuning method are available in [18]. The calculated observer gain is:

$$
L=\left[\begin{array}{ccc}
-0.0097 & -0.0097 & -0.0197 \\
0.1743 & 0.0311 & -0.0056 \\
0.0141 & -0.0004 & -0.4141 \\
-0.2254 & -0.0056 & 4.2290
\end{array}\right]
$$

For the 3-DOF vehicle model described by Equations (1), (2), (3), (4), (5), (6), (7), (8), (9), (10), (11), (12), (13), (14), $(15),(16),(17),(18),(19),(20),(21)$ and equipped with the chosen sensor set (Equations (22), (23), (24), the Luenberger observer is designed and implemented in Matlab/Simulink and CarSim. Figure 3 illustrates the Simulink model of the observer.

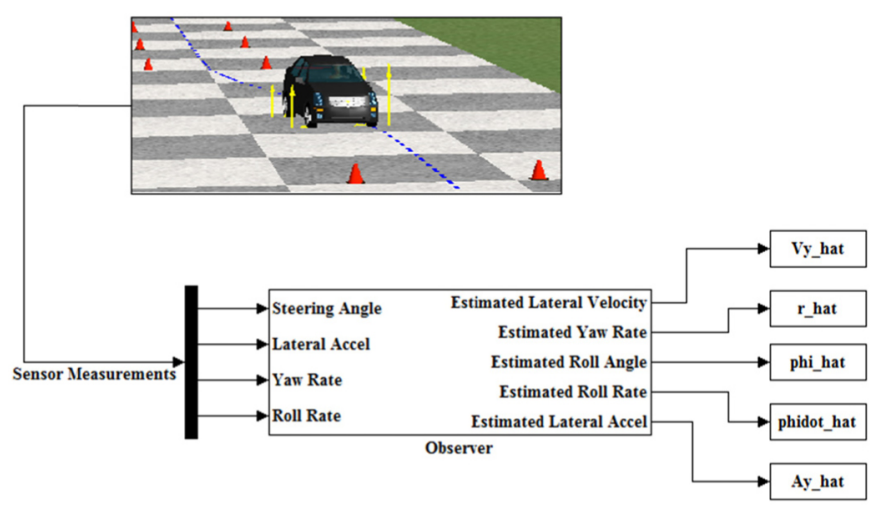

Figure 3. Implementation of the observer in Simulink and CarSim

Performance of the observer of the states is evaluated with a double-lane-change maneuver. Input steering angle and the sensory signals from CarSim are fed to the observer and the estimated states are extracted. Simulation results for estimation of the lateral velocity and roll angle are illustrated in Figure 4 and Figure 5, respectively. The estimation results are compared with the corresponding results from CarSim.

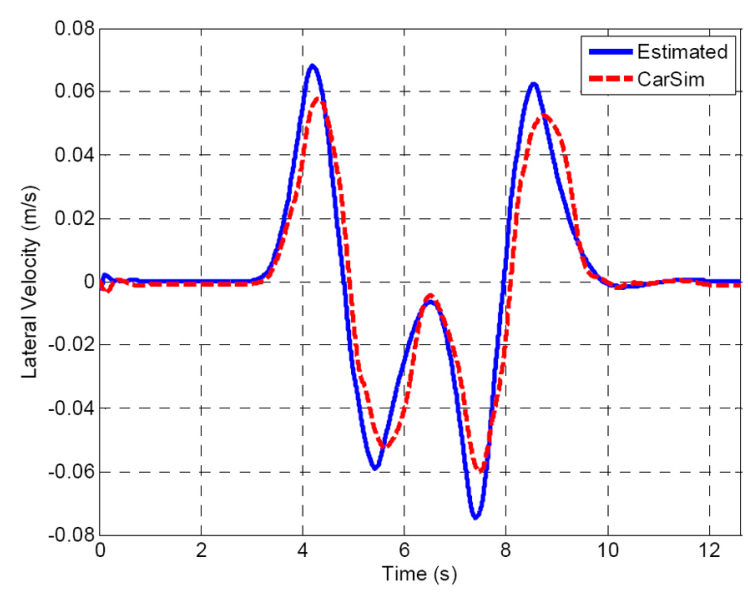

Figure 4. Estimation of lateral velocity 


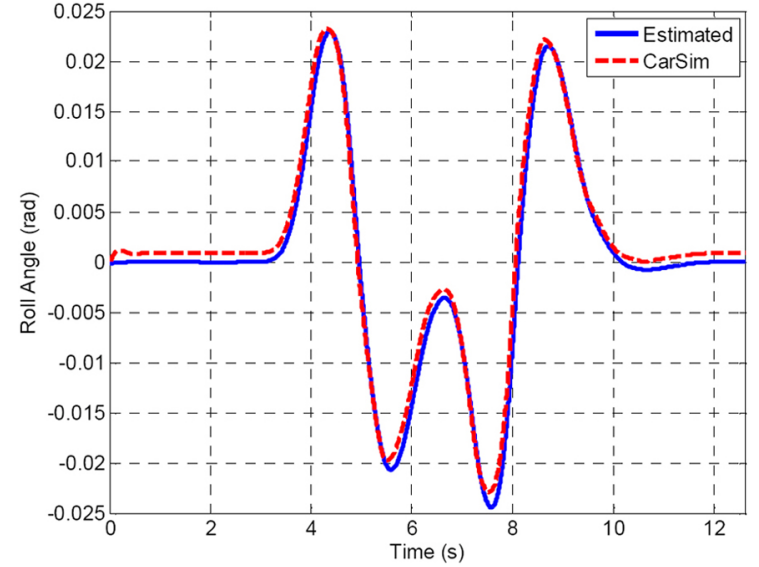

Figure 5. Estimation of roll angle

The results verify the desirable performance of the estimator despite modeling uncertainties and simplicity of the model.

\section{Effects of Faulty Sensory Measurements on Observer Performance}

This sub-section investigates the performance of the estimator in the presence of sensor faults. As an example, it is assumed that the lateral acceleration signal is generating false measurements (zero signal) after the 3rd second of the maneuver. The situation is simulated in CarSim and the same estimation approach as discussed for normal conditions is carried out to analyze the performance of the observer. Simulation results for estimation of lateral velocity and roll angle are illustrated in Figure 6 and Figure 7, respectively.

According to the results, although only one of the available three sensors is generating false information, the observer fails to accurately estimate the states. It should be noted that such an issue is not dependent on the choice of the observer because even complex-nonlinear observers are prone to such difficulties.

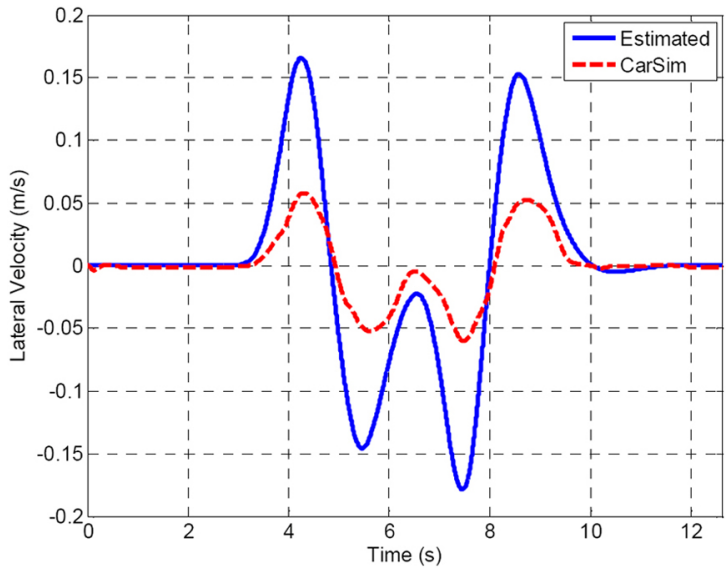

Figure 6. Estimation of lateral velocity using faulty acceleration signal

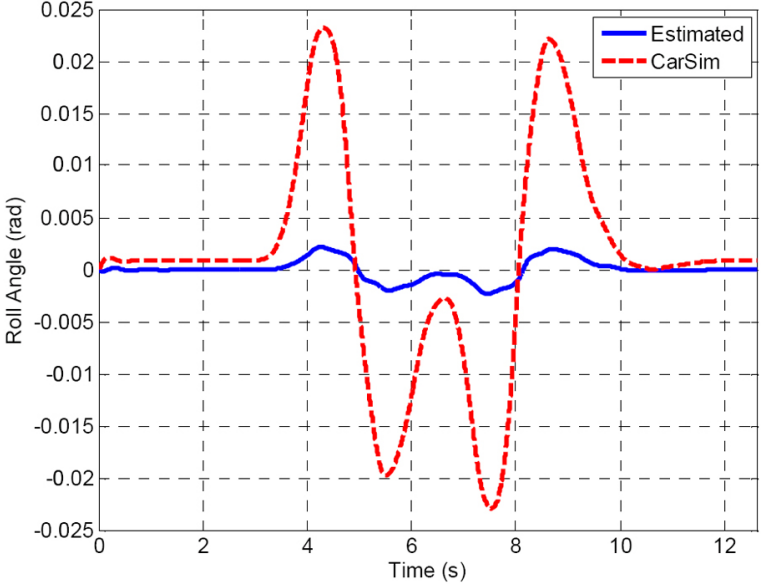

Figure 7. Estimation of roll angle using faulty acceleration signal

This implies a necessity of a fault-tolerant estimation method that can reliably estimate the state in the presence of possible sensor faults.

\section{Design of Fault-Tolerant Adaptive Bank of Observers}

According to the literature, a system is called faulttolerant if it can successfully overcome the difficulties that arise from the occurrence of a fault. In this paper, an adaptive fault-tolerant bank of observers is developed for fault-tolerant estimation of the vehicle states. Overall scheme of the method is illustrated in Figure 8.

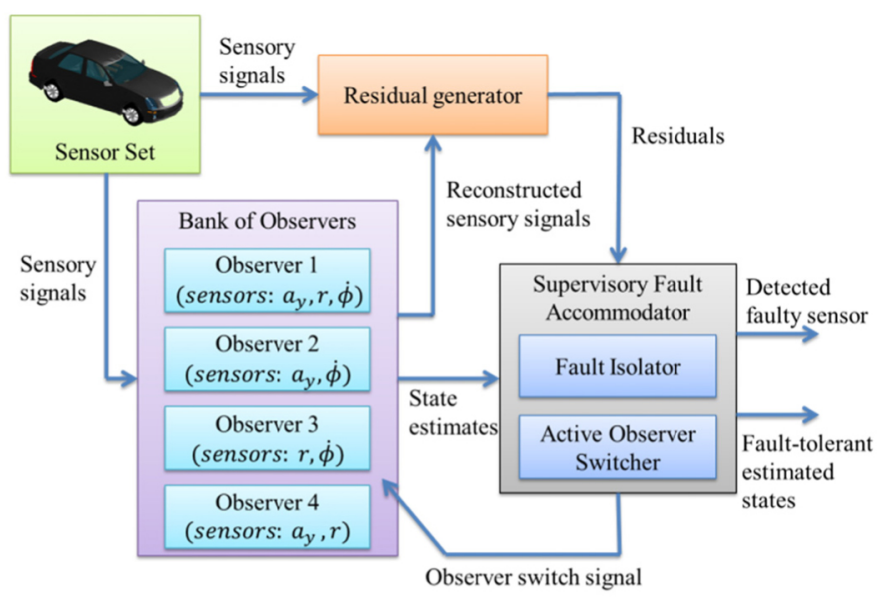

Figure 8. Adaptive fault-tolerant bank of observers

Detailed description of the blocks used in this model is presented as follows:

\section{Bank of Observers Block}

This block contains four Luenberger observers which are designed and tuned the same way as discussed in Sub-section A. The main difference is the insensitivity of each of the observers to one of the sensory signals which allows 
exploitation of the available sensor redundancy to accommodate the faults. Preference of each observer to serve as the active observer is determined with respect to its optimality in terms of sensor configuration, as discussed in Section III. The LQR method is applied to tune the gain for each of the observers. The primary observer (observer 1 ) acts as the main observer using all of the available measurement signals and was described in Sub-section A. As mentioned before, the sensor set used in this observer has the highest observability rank among the cases discussed in Section III.

For the other three observers, the measurement equation (2) is different. Details for these observers can be summarized as:

- Observer 2:

Sensors: lateral acceleration and roll rate

Matrices in the measurement equation (C and D):

$$
\begin{gathered}
C_{2}=\left[\begin{array}{cccc}
a_{11} & a_{12} & a_{13} & a_{14} \\
0 & 0 & 0 & 1
\end{array}\right] \\
D_{2}=\left[\begin{array}{c}
b_{11} \\
0
\end{array}\right]
\end{gathered}
$$

Observer gain:

$$
L_{2}=\left[\begin{array}{cc}
-0.5238 & -0.0197 \\
0.1746 & -0.0056 \\
0.0140 & -0.4141 \\
-0.2255 & 4.2290
\end{array}\right]
$$

Relative rank (with respect to sensor configuration): 2

- Observer 3:

Sensors: yaw rate and roll rate

Matrices in the measurement equation (C and D):

$$
\begin{gathered}
C_{3}=\left[\begin{array}{llll}
0 & 1 & 0 & 0 \\
0 & 0 & 0 & 1
\end{array}\right] \\
D_{3}=\left[\begin{array}{l}
0 \\
0
\end{array}\right]
\end{gathered}
$$

Observer gain:

$$
L_{3}=\left[\begin{array}{cc}
-0.0121 & -0.0074 \\
0.0318 & -0.0077 \\
-0.0003 & -0.4142 \\
-0.0077 & 4.2453
\end{array}\right]
$$

Relative rank (with respect to sensor configuration): 3

- Observer 4:

Sensors: lateral acceleration and yaw rate

Matrices in the measurement equation (C and $\mathrm{D})$ :

$$
C_{4}=\left[\begin{array}{cccc}
a_{11} & a_{12} & a_{13} & a_{14} \\
0 & 1 & 0 & 0
\end{array}\right]
$$

$$
D_{4}=\left[\begin{array}{c}
b_{11} \\
0
\end{array}\right]
$$

Observer gain:

$$
L_{4}=\left[\begin{array}{cc}
-0.5236 & -0.0096 \\
0.1743 & 0.0311 \\
0.0159 & -0.0003 \\
-0.2069 & -0.0074
\end{array}\right]
$$

Relative rank (with respect to sensor configuration): 4

The bank of observers receives sensory signals and generates the estimated states. The supervisory faultaccommodator block selects the active observer from the bank, depending on which sensor is diagnosed as faulty.

\section{Residual Generator Block}

The residual generator block receives the estimated states from the bank of observers, and the sensory information from CarSim (or the actual vehicle sensors in the case of real application). This block simply compares the observer outputs and real measured outputs while transferring the residuals, i.e. difference between the measured and reconstructed signal by the observer, to the supervisory fault accommodator block.

\section{Supervisory Fault Accommodator Block}

This block receives the generated residual and compares them to the acceptable disturbance bound for each of the sensors. The bounds are determined based on the normal disturbances observed during operation of the observer for normal (fault-free) situations. In fact, the bounds determine the acceptable discrepancy between the measured and estimated quantities which originates from modeling uncertainties and noises. In this paper, the bounds are set as:

- Lateral acceleration bound $\left(\hat{a}_{y}-a_{y}\right): \pm 0.8\left(\mathrm{~m} / \mathrm{s}^{2}\right)$

- Yaw rate bound $(\hat{r}-r): \pm 0.04(\mathrm{rad} / \mathrm{s})$

- Roll rate bound $(\hat{\dot{\phi}}-\dot{\phi}): \pm 0.04(\mathrm{rad} / \mathrm{s})$

The supervisory fault accommodator block switches the active observer if the absolute value of any of the residuals for a sensor used by that observer exceeds its normal disturbance bound. Such values demonstrate that a factor other than the modeling uncertainty or noises is causing a large difference between the estimated and the measured quantities. This judgement is valid if the observer is efficiently designed for normal conditions, which was demonstrated for the present case study in the previous subsections. The algorithm assigns a value of 1 as a fault index for the failed sensor to show that the sensor is malfunctioning and should be excluded from the estimation process.

The supervisory block reconfigures the adaptive bank of the observers block by switching the active observer to the 
one that is insensitive to the detected faulty sensor and has the highest relative rank compared to the other sets.

\section{Simulation Results for Fault-Tolerant Estimation of the States}

In order to demonstrate the effectiveness of the proposed scheme, the same simulation as described in Sub-section B is repeated here. The previous single observer method is replaced with the fault-tolerant estimation scheme. Similarly, after the 3rd second of the maneuver, failure of the lateral acceleration signal is simulated and the estimated results are compared with CarSim measurement signals. Figure 9 shows a successful detection of the failed sensor by the algorithm. As described before, a fault index value of 1 represents detection of a fault on the sensor at around 3.8 second.
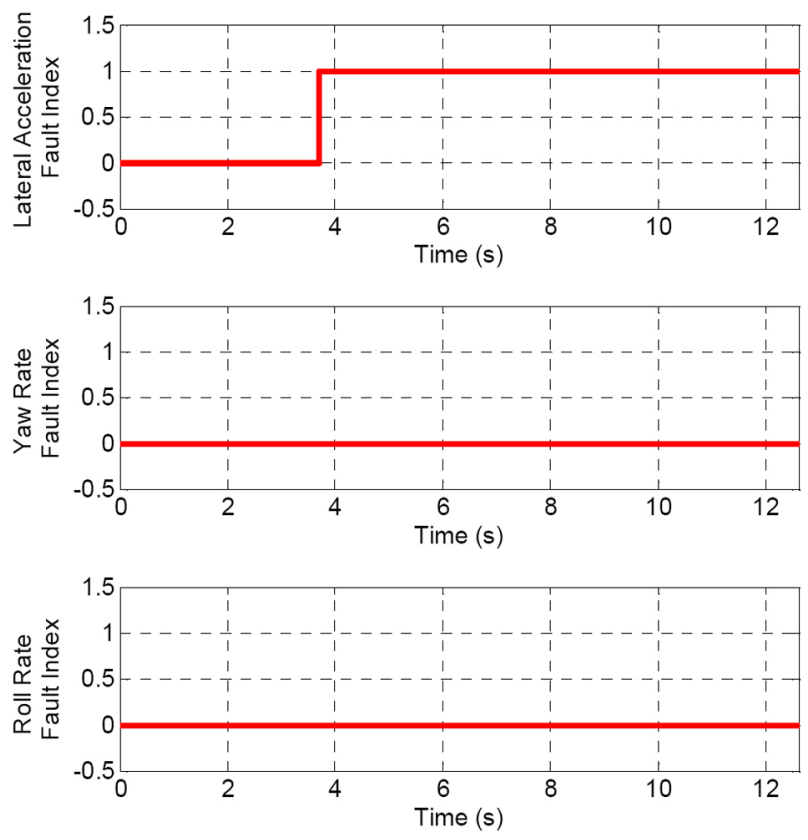

Figure 9. Detection of the faulty sensor

Simulation results for estimation of the states and reconstruction of the measurements are illustrated in Figures $\underline{10}, \underline{11}, \underline{12}, \underline{13}, \underline{14}$. Reconstruction of the sensory measurements is performed using the estimated states and the measurement equation (22) and is carried out in real-time with the observer scheme.

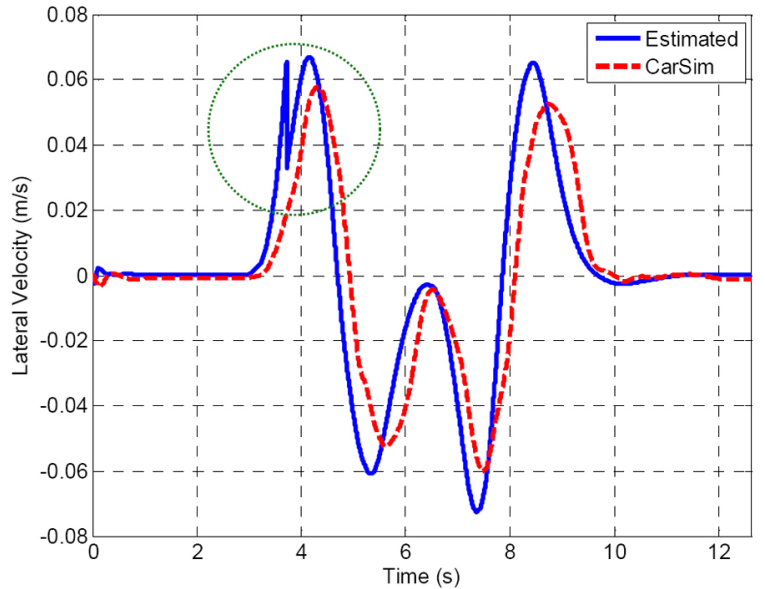

Figure 10. Fault-tolerant estimation of lateral velocity

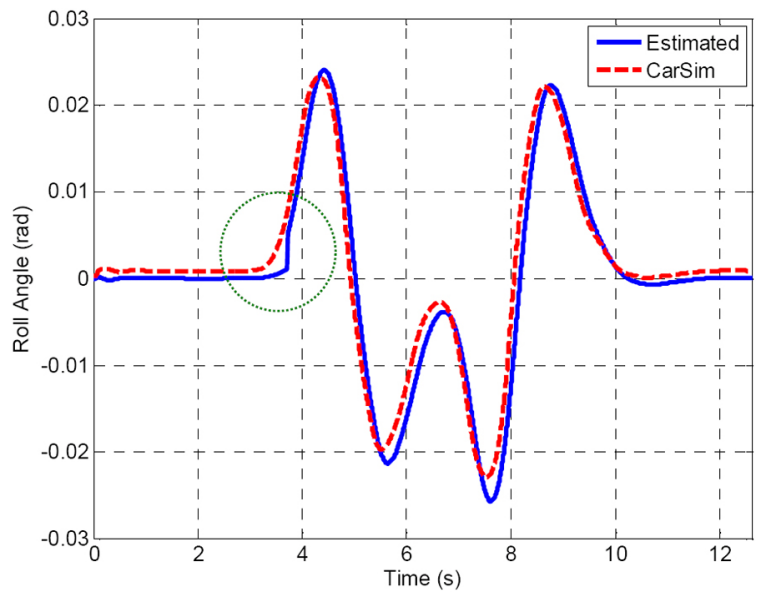

Figure 11. Fault-tolerant estimation of roll angle

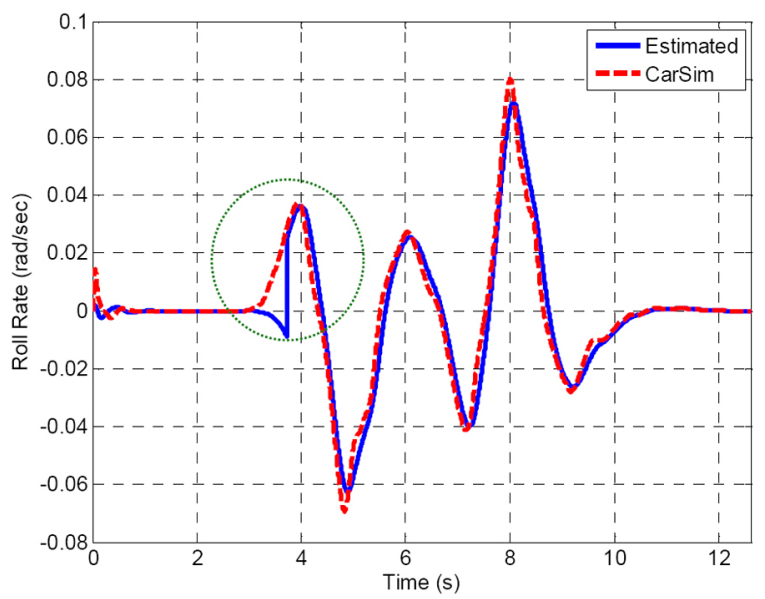

Figure 12. Fault-tolerant reconstruction of roll rate 


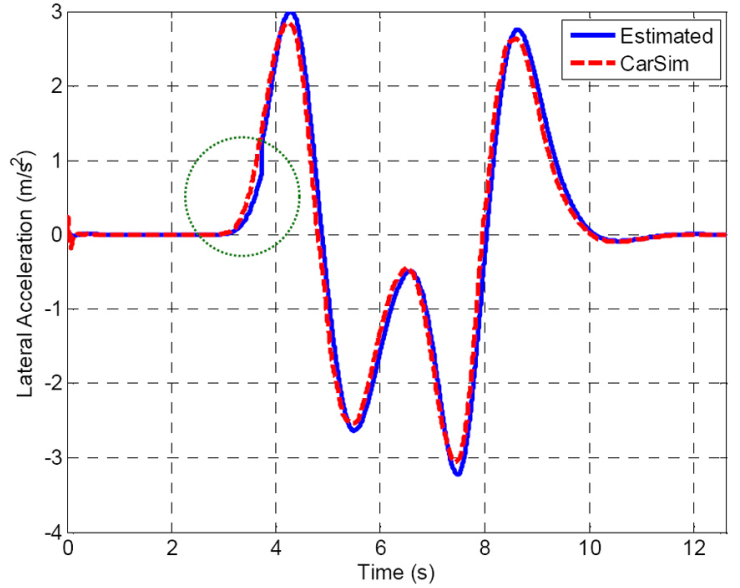

Figure 13. Fault-tolerant reconstruction of lateral acceleration

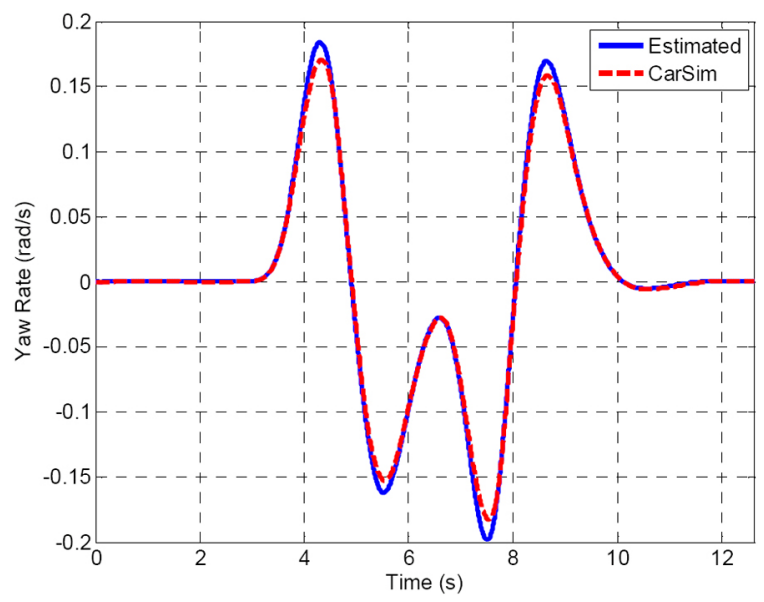

Figure 14. Fault-tolerant reconstruction of yaw rate

The marked regions of the figures show how the algorithm successfully prevents the estimates from diverging from their real values. Such prevention is achieved through appropriate reconfiguration of the observer block, and exclusion of the faulty sensor from the estimation process. Similar performance is observed for yaw rate and roll rate sensor failures but the results are not demonstrated here for brevity.

It should be noted that the case study presented here considers single sensor failure (one sensor failure at a time). However, the approach is general and multiple sensor failures can be detected by increasing the number of observers. As an example, using seven observers in the bank with the sensor sets described in Table 1, multiple failures (two-sensors at a time) are recoverable with the proposed approach. Although this will increase the computational cost, but the algorithm is not highly demanding due to use of the simple vehicle model and linear observers. Moreover, the capable processing units in modern vehicles enable real-time implementation of the proposed approach even for the cases that nonlinear observers (e.g. Extended Kalman Filter) and high-DOF vehicle models are used in the proposed structure.

\section{SUMMARY/CONCLUSIONS}

This paper investigated model-based optimal sensor configuration and fault-tolerant state estimation for vehicle applications. Rather than using a 2-DOF vehicle model that neglects the effects of lateral load transfer and other rollmotion-related effects, a 3-DOF vehicle handling model was formulated and simulated. Comparison of the simulated results with a sophisticated CarSim vehicle model demonstrated desirability of the model despite its simplicity.

Using the developed model, observability and optimal sensor configuration for estimation of the vehicle handling states was investigated. Several observability measures were utilized and the best sensor configuration was determined.

The optimal sensor set was used for estimation of vehicle handling states and the results were compared with CarSim to verify desirable performance of the estimator in normal (fault-free) conditions. In order to study the effects of possible sensor failures on the estimation performance, failure of the accelerometer was simulated and the estimation performance was analyzed. Although the estimator was exploiting all of the available measurements, the injected fault had a significant effect on the estimation. To overcome this issue, an adaptive bank of observers was designed using the vehicle model and the results of the observability analysis. Performance of the fault-tolerant estimation scheme was investigated through simulations and comparisons with CarSim. Simulation results demonstrate desirable estimation of the states even in the conditions that a sensor was transmitting false information to the estimator.

The proposed approach can be recruited for online detection of sensor failures, fault-tolerant estimation of vehicle states, and also reconstruction of sensory signals for vehicle stability and control applications.

\section{REFERENCES}

1. Muller, P. C., Weber, H. I., "Analysis and Optimization of Certain Qualities of Controllability and Observability for Linear Dynamical Systems," Automatica, 8(3): 237-246, 1972, doi: 10.1016/0005-1098(72)90044-1.

2. Chamseddine, A., Noura, H., Raharijaona, T., "Optimal Sensor Network Design for Observability of Complex Systems," Proceedings of the American Control Conference, New York, USA, pp. 1705-1710, 2007, doi: 10.1109/ACC.2007.4282689.

3. Sumana, C., Venkateswarlu, Ch., "Optimal Selection of Sensors for State Estimation in a Reactive Distillation Process," Journal of Process Control, 19(6):1024-1035, 2009, doi: 10.1016/j.jprocont.2009.01.003.

4. Nakhaeinejad, M., Bryant, M. D. "Observability Analysis for ModelBased Fault Detection and Sensor Selection in Induction Motors," Meas. Sci. Technol., 22, 2011, doi: 10.1088/0957-0233/22/7/075202.

5. Letellier, Ch., Aguirre, L. A., "Investigating Nonlinear Dynamics From Time Series: The Influence of Symmetries and the Choice of Observables," Chaos, 12(3):549-558, 2002, doi: 10.1063/1.1487570.

6. Stéphant, J., Charara, A., Meizel, D., "Evaluation of a Sliding Mode Observer for Vehicle Sideslip Angle," Control Engineering Practice, 7(15):803-812, 2007, doi: 10.1016/j.conengprac.2006.04.002.

7. Arat, M., Singh, K., and Taheri, S., "An Adaptive Vehicle Stability Control Algorithm Based on Tire Slip-Angle Estimation," SAE Technical Paper 2012-01-2016, 2012, doi: 10.4271/2012-01-2016.

8. Metallidis, P., Verros, G., Natsiavas, S., 2003, "Fault Detection and Optimal Sensor Location in Vehicle Suspensions," Journal of Vibration and Control, 9:337-359, 2003, doi: 1 10.1177/107754603030755. 
9. Kim, J., and Lee, H., "Sensor Fault Detection and Isolation Algorithm for a Continuous Damping Control System," Proceedings of the Institution of Mechanical Engineers, Part D: Journal of Automobile Engineering, $\quad 0954407011404493, \quad 2011, \quad$ doi: 10.1177/0954407011404493.

10. Fischer, D., Börner, M., Schmitt, J., "Fault Detection for Lateral and Vertical Vehicle Dynamics," Control Engineering Practice, 15(3): 315-324, 2007, doi: 10.1016/j.conengprac.2006.05.007.

11. Nishio, A., Tozu, K., Yamaguchi, H., Asano, K., Amano, Y., "Development of Vehicle Stability Control System Based on Vehicle Sideslip Angle Estimation," SAE Technical Paper 2001-01-0137, 2001, doi: $10.4271 / 2001-01-0137$.

12. Piyabongkarn, D., Rajamani, R., Grogg, J. A., "Development and Experimental Evaluation of a Slip Angle Estimator for Vehicle Stability Control," IEEE Transactions on Control Systems Technology, 17(1): 78-88, 2009, doi: 10.1109/TCST.2008.922503.

13. Guvenc, B. A Guvenc, L., and Karaman, S., "Robust Yaw Stability Controller Design and Hardware-in-the-Loop Testing for a Road Vehicle," IEEE Transactions on Vehicular Technology, 58(2): 555-5712009, 2009, doi: 10.1109/TVT.2008.925312.

14. Canale, M., Fagiano, L., Ferrara, A., "Comparing Internal Model Control and Sliding-Mode Approaches for Vehicle Yaw Control," IEEE Transactions on Intelligent Transportation Systems, 10(1):31-41, 2009, doi: 10.1109/TITS.2008.2006772.

15. Cong Geng, Mostefai, L., Denai, M., "Direct Yaw-Moment Control of an in-Wheel-Motored Electric Vehicle Based on Body Slip Angle Fuzzy Observer," IEEE Transactions on Industrial Electronics, 56(5): 1411-1419, 2009, doi: 10.1109/TIE.2009.2013737.

16. Jazar R. N., "Vehicle Dynamics Theory and Application", Springer, ISBN-13: 978-0387742434, 2008.

17. Singh, A.K, Hahn, J., "Determining Optimal Sensor Locations for State and Parameter Estimation for Stable Nonlinear Systems", Industrial and Engineering Chemistry Research, 44 (5):5645-56592005, 2005, DOI: 10.1021/ie040212v.

18. Ogata, K., "Modern Control Engineering", Prentice Hall, ISBN-13: 978-0136156734, 2009

\section{ACKNOWLEDGMENTS}

The authors would like to acknowledge the financial support of the Automotive Partnership Canada, Ontario Research Fund.

\section{DEFINITIONS/ABBREVIATIONS}

CG - center of gravity

$\mathbf{C}_{\mathbf{t}}$ - roll damping coefficient

$\mathbf{C}_{\boldsymbol{\alpha} \mathbf{f}}, \mathbf{C}_{\boldsymbol{\alpha} \mathbf{r}}$ - cornering stiffness of the wheels (front, rear)

$\mathbf{C}_{\boldsymbol{\gamma} \mathbf{f}}, \mathbf{C}_{\boldsymbol{\gamma} \mathbf{r}}$ - camber stiffness (front, rear)

DOF - degree of freedom

$\mathbf{F}_{\mathbf{y f l}}, \mathbf{F}_{\mathbf{y f r}}, \mathbf{F}_{\mathbf{y r l}}, \mathbf{F}_{\mathbf{y r r}}-$ lateral tire forces

g - gravity constant

$\mathbf{h}^{\prime}$ - distance between the roll center and center of gravity

$\mathbf{I}_{\mathbf{Z}}$ - yaw moment of inertia

$\mathbf{K}_{\mathbf{t}}$ - roll stiffness coefficient

$\mathbf{K}_{\mathrm{fSBR}}, \mathbf{K}_{\mathrm{rSBR}}$ - steer by roll coefficients (front, rear)

$\mathbf{K}_{\mathrm{fCBR}}, \mathbf{K}_{\mathrm{rCBR}}$ - camber by roll coefficients (front, rear)

$\mathbf{K}_{\mathbf{f S C B R}}, \mathbf{K}_{\mathbf{r S C B R}}$ - scrub by roll coefficients (front, rear)

L - wheelbase

$\mathbf{L}_{\mathbf{f}}, \mathbf{L}_{\mathbf{r}}$ - horizontal distance between the center of gravity of the vehicle and the front and rear axles, respectively

m - vehicle mass $\mathbf{m}_{\mathbf{s}}$ - mass of the sprung mass

RC - roll center

T - track width

u - longitudinal velocity

xyz - body-fixed coordinate system 